\title{
The Effects and Mechanism of Leader's Emotional Intelligence on Team Innovation Performance
}

\author{
Shuhong Wang* \\ Zhongnan University of Economics and Law \\ Wuhan, China
}

\author{
Menmei Wu \\ Zhongnan University of Economics and Law \\ Wuhan, China
}

\author{
Wenyue Pan \\ Zhongnan University of Economics and Law \\ Wuhan, China
}

\begin{abstract}
This paper intends to study the influence and mechanism of leader's emotional intelligence on the team innovation performance using a group-level design with empirical research method. The study shows that leader's emotional intelligence, leader--member exchange and team psychological safety have significant positive impacts on team innovation performance. Meanwhile, leader-member exchange and team psychological safety have partial mediating effects on the relationship between leader's emotional intelligence and team innovation performance. Several methods are suggested to enhance the team innovation performance.
\end{abstract}

Keywords-Leader's Emotional Intelligence; leader-member Exchange; team Psychological Safety; team Innovation Performance

\section{INTRODUCTION}

Back in early 20th century, economist Joseph Schumpeter, author of The Theory of Economic Development-the first work of innovation theory, has demonstrated the great impact of innovation. With the advent of the knowledge-economy era in 21th century, enhancing the ability of innovation has become the strategic choice of many organizations. Employees' value is not only reflected in their work force, but also in their innovative ideas [1] which could be transformed into products and services. Team division and cooperation is replacing individuals as the main executive unit. How to improve team innovation performance has become a widely concerned problem.

All innovation performance is conducted by employees. Many scholars believe that leader's emotional intelligence is conducive to establishing a positive working environment and can effectively predict employee performance [2]. However, little research has been done on the relation between leader's emotional intelligence and team innovation performance. Compared to less hierarchical culture, Chinese culture contains more power distance and employees are more terrified when associating with leaders, so the requirement of leader's emotional intelligence is stricter [3]. It is of great theoretical and practical significance to research how leaders identify, control and manage emotions and promote team innovation performance.

The Project supported by Humanities and Social Sciences of Ministry of Education Planning Fund (Grant NO.19YJA630081)

\section{THEORY AND HYPOTHESES}

\section{A. Definitions of the Constructs}

\section{1) Emotional Intelligence}

At present, the representative views about emotional intelligence (EI) include the ability model proposed by Salovey and Mayer, and the mixed model, which is more comprehensive, proposed by Bar-On and Goleman. Salovey and Mayer put EI into the category of intelligence and believe it is the ability of processing emotional information. The model claims that EI contains four types of abilities: perceiving emotions, using emotions, understanding emotions and managing emotions [4].

\section{2) Leader-member Exchange}

The leader-member exchange theory (LMX) was proposed firstly by Graen and Cashman who defined it as "a relationship-based social exchange between leaders and followers" [5]. The theory suggests that leaders have limited energy and time, so they do not treat each subordinate the same. Leaders will divide subordinates into "in-group" and "out-group" based on the extent of closeness, and have different attitude towards them. In return, the job of employees will be different.

\section{3) Team Psychological Safety}

The conception of team psychological safety (TPS) was proposed by Edmondson. It is considered to be a shared belief that the team is safe for risk taking [6]. Under the influence of TPS, team members dare to take interpersonal risks, point out others' mistakes and argue about a point of view even in the absence of TPS conditions.

\section{4) Team Innovation Performance}

The "task-contextual performance" model is the most common model of assessing job performance in the West but it does not define innovation very well. Han $\mathrm{Yi}$ and other scholars put forward a four-factor model including task performance, contextual performance, learning performance and innovation performance [7].

Team innovation performance (TIP) contains not only introducing and applying new ideas and procedures, but also the overall performance of innovative achievement. 


\section{B. Hypotheses}

1) Leader's Emotional Intelligence and Team Innovation Performance

Goleman applied EI to working scene for the first time in 1998. He pointed out that compared to leaders of average performance; nearly $90 \%$ of the difference of star performers was attributable to EI factors [8]. Although leadership style will influence innovation performance [9], Zhou and George believe that leaders' $\mathrm{EI}$ is the source whether they support employees' innovation [10]. Leaders with higher EI are better at identifying and controlling team members' emotions and are more sensitive to employees' innovative intention so that they will show a higher level of support, hence affect TIP. Meanwhile, employees with positive emotional state are more likely to have better innovation performance. Therefore a hypothesis is proposed:

Hypothesis 1: Leader's EI is positively related to TIP.

2) Leader's Emotional Intelligence, Leader-member Exchange and Team Innovation Performance

The theoretical basis of LXM is social exchange theory, according to which the interaction between people is a social exchange process including material and immaterial exchange. When managing the relation of team members, leaders with high EI will not only provide them with substantial support like capital and equipment, but also with personalized care and emotional support, thus developing a high-quality LMX relationship in which members believe that they are valuable. According to the principle of reciprocity, in order to repay supervisors' recognition and support, team members will have sense of responsibility for organizational interests and goals, and reward organization by innovation. In the high-quality LMX relationship, leaders and members will form a benign interaction relationship. Therefore the following hypotheses are proposed:

Hypothesis 2: Leader's EI is positively related to LMX.

Hypothesis 3: LMX is positively related to TIP.

Hypothesis 4: LMX mediates the relationship between leader's EI and TIP.

3) Leader's Emotional Intelligence, Team Psychological Safety and Team Innovation Performance
In team innovation, pointing out others' mistakes face to face and presenting innovative ideas may lead to interpersonal relationship risks. Low psychological safety causes anxiety about innovation failure and the negative impact on their image, status or job [11] and will eventually hinder team innovation. Leaders with higher EI are more sensitive to perceiving emotions of team members and enhance their' psychological safety, therefore effectively promote innovation [12]. TPS creates a "safe atmosphere" in which team members are less concerned about interpersonal risks and are more likely to focus on solving problems creatively [13].

Hypothesis 5: Leader's EI is positively related to TPS.

Hypothesis 6: TPS is positively related to TIP.

Hypothesis 7: The relationship between leader's EI and TIP is mediated by TPS.

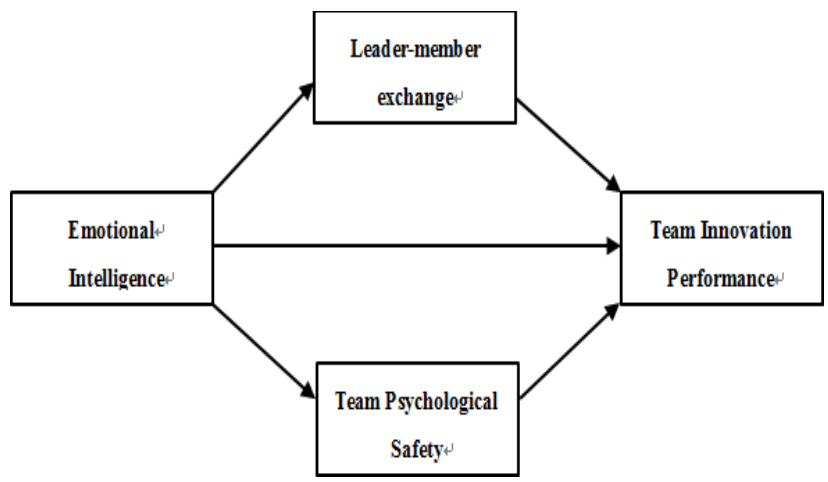

Fig. 1. The research framework of this paper

\section{EMPIRICAL RESEARCH}

\section{A. Sample Selection and Data Gathering}

In order to reduce common method variance, we adopt heterologous data and paired sample. A paired questionnaire survey was conducted in 18 representative industries in Beijing, Wuhan and Guangzhou. Altogether, 106 valid questionnaires were gathered. The sample distribution characteristics are shown in TABLE I.

TABLE I. STATISTIC OF SAMPLE DISTRIBUTION(N=106 / 532)

\begin{tabular}{|c|c|c|c|c|c|}
\hline \multirow{2}{*}{\multicolumn{2}{|c|}{ Variable }} & \multicolumn{2}{|c|}{ Frequency } & \multicolumn{2}{|c|}{ Ratio (\%) } \\
\hline & & Manager & Subordinate & Manager & Subordinate \\
\hline \multirow{2}{*}{ Gender } & Male & 91 & 303 & 84.26 & 56.95 \\
\hline & Female & 17 & 229 & 15.74 & 43.05 \\
\hline \multirow{4}{*}{$\begin{array}{l}\text { Age } \\
\text { (year) }\end{array}$} & $20-28$ & 8 & 72 & 7.41 & 13.53 \\
\hline & $29-35$ & 43 & 215 & 39.81 & 40.41 \\
\hline & $36-45$ & 32 & 152 & 29.63 & 28.57 \\
\hline & 46 or older & 25 & 93 & 23.15 & 17.49 \\
\hline \multirow{5}{*}{ Education } & Below high school & 3 & 18 & 2.78 & 3.39 \\
\hline & Secondary specialized school/high school & 8 & 40 & 7.41 & 7.52 \\
\hline & Junior college & 17 & 98 & 15.74 & 18.42 \\
\hline & Bachelor's degree & 67 & 343 & 62.04 & 64.47 \\
\hline & Master's degree/Ph.D. & 13 & 33 & 12.03 & 6.20 \\
\hline
\end{tabular}




\section{B. Variable Measurement}

EI was measured by the WLEIS scale (Wong and Law Emotional Intelligence Scale) [14] consisting of four dimensions including self-emotional appraisal, others-emotional appraisal, use of emotion and regulation of emotion with 16 items. LMX was measured by the scale of Graen and Uhl-Bien [5] including 7 items. Measurement of TPS adopted the scale of Edmondson [6] with 5 items. Measurement of team innovation performance used scale of Guo Wei and Li Yanping [15] including 10 items. Except for demographic variables, others adopted a five-point Likert scale.

\section{Reliability and Validity Test of the Scale}

\section{1) Reliability Test}

Internal consistency coefficient was used to analyze the reliability. $\alpha$ coefficient of the four scales are $0.897,0.870$, 0.856 and 0.915 respectively, which are all above 0.7 . It can be considered that the sample data is reliable. In addition, the four dimensions' $\alpha$ coefficient of EI scale are $0.810,0.819,0.804$ and 0.855 respectively.

The research object of this study is team. However, the data of dependent variables and mediators originates from answers of team members. Therefore, it is necessary to integrate individual-level data into team level. The premise of data integration is that team members have high cognitive consistency for the items, and there is enough diversity between different teams. This paper adopted Rwg, ICC (1) and ICC (2) to test whether the indicators meet the standards. The three indicators are usually required to be above $0.12,0.70$ and 0.70 respectively. The indicators in TABLE II all exceed the empirical standards, implying that the participants in the same team have high consistency and it is feasible to aggregate data from individual level to team level.

TABLE II. THE ICC1, ICC2 AND $\Gamma$ WG OF VARIABLES

\begin{tabular}{|c|c|c|c|c|c|}
\hline Variable & ICC1 & ICC2 & $\boldsymbol{\gamma}$ wg & T00 & $\boldsymbol{\sigma 2}$ \\
\hline LMX & 0.176 & 0.727 & 0.842 & 0.122 & 0.320 \\
\hline TPS & 0.210 & 0.850 & 0.782 & 0.148 & 0.558 \\
\hline CX & 0.127 & 0.915 & 0.820 & 0.082 & 0.226 \\
\hline
\end{tabular}

2) Validity Test

This study used structural equation modeling (SEM) to conduct confirmatory factor analysis. According to TABLE III, NNFI, GFI and CFI are all greater than 0.90, RMSEA is close to 0.08 and $\chi^{2} / \mathrm{df}$ is close to 3 , indicating that the model have fitted the data very well and each questionnaire has high structural validity and discriminant validity.

TABLE III. THE RESULT OF STRUCTURAL VALIDITY ANALYSIS

\begin{tabular}{|c|c|c|c|c|c|c|c|}
\hline Questionnaire structure & $\boldsymbol{\chi}^{\mathbf{2}}$ & $\mathbf{d f}$ & $\boldsymbol{\chi}^{\mathbf{2}} / \mathbf{d f}$ & GFI & NNFI & CFI & RMSEA \\
\hline EI(four factors) & 262.050 & 98 & 2.670 & 0.950 & 0.920 & 0.950 & 0.067 \\
\hline $\begin{array}{c}\text { LMX } \\
\text { (four factors) }\end{array}$ & 238.970 & 84 & 2.840 & 0.930 & 0.910 & 0.930 & 0.066 \\
\hline $\begin{array}{c}\text { TPS } \\
\text { (single factor) }\end{array}$ & 30.352 & 14 & 2.168 & 0.91 & 0.921 & 0.920 & 0.059 \\
\hline $\begin{array}{c}\text { CX } \\
\text { (single factor) }\end{array}$ & 28.032 & 8 & 3.504 & 0.910 & 0.900 & 0.920 & 0.088 \\
\hline $\begin{array}{c}\text { four factors: } \\
\text { EI; LMX; TPS; CX }\end{array}$ & 217.120 & 59 & 3.68 & 0.910 & 0.910 & 0.920 & 0.069 \\
\hline
\end{tabular}

\section{3) Descriptive Statistics}

According to TABLE IV, the correlation coefficients of all variables reach a significant level, indicating a positive correlation between the four variables.
TABLE IV. DESCRIPTIVE STATISTICAL ANALYSIS AND VARIABLE CORRELATION MATRIX

\begin{tabular}{|c|c|c|c|c|c|}
\hline Variable & $\mathbf{M}$ & $\mathbf{S D}$ & $\mathbf{1}$ & $\mathbf{2}$ & $\mathbf{3}$ \\
\hline EI & 4.180 & 0.640 & & & \\
\hline LMX & 4.130 & 0.580 & $0.365^{* *}$ & & \\
\hline TPS & 3.750 & 0.760 & $0.272^{* *}$ & $0.482 * *$ & \\
\hline CX & 3.480 & 0.750 & $0.289^{* *}$ & $0.377^{* *}$ & $0.412^{* *}$ \\
\hline
\end{tabular}

\section{Hypothesis Testing}

SEM was used to test the mediating effects of the mediators Four variables were put together for modeling verification. The test results are shown in TABLE V.

TABLE V. THE RESULT OF REgRESSION ANALYSIS OF LMX AND TPS

\begin{tabular}{|c|c|c|c|c|c|c|c|}
\hline \multicolumn{2}{|c|}{ Structural model } & $\chi^{2}$ & df & $\chi^{2} / \mathbf{d f}$ & NNFI & CFI & RMSEA \\
\hline Partial mediation model & $\mathrm{EI} \rightarrow \mathrm{LMX} \rightarrow \mathrm{CX} \& \mathrm{EI} \rightarrow \mathrm{CX}$ & 203.422 & 62 & 3.2810 & 0.910 & 0.930 & 0.074 \\
\hline Full mediation model & $\mathrm{EI} \rightarrow \mathrm{LMX} \rightarrow \mathrm{CX}$ & 389.590 & 65 & 5.9936 & 0.550 & 0.600 & 0.272 \\
\hline
\end{tabular}

According to TABLE $\mathrm{V}$, the fitting degree of partial mediation model is generally higher than that of full mediation model. $\chi^{2} / \mathrm{df}$, NNFI, CFI and RMSEA of partial mediation model of LMX is 3.2810, 0.910, 0.930 and 0.074 respectively. $\chi^{2} / \mathrm{df}, \mathrm{NNFI}, \mathrm{CFI}$ and RMSEA of partial mediation model of team psychological safety is 3.0245, 0.920, 0.940 and 0.067 respectively. The fit index is ideal. LMX and TPS are both 
acceptable, and the partial mediation model is acceptable as well.

According to Figure 2, leaders' emotional intelligence has both direct and indirect significant effects on team innovation performance, and leader-member exchange and team psychological safety have partial mediating effects between them. Meanwhile leader-member exchange has effects on team psychological safety.

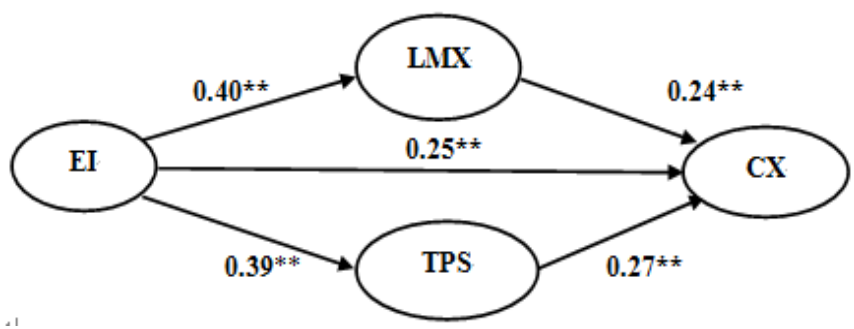

Fig. 2. Conceptual model and estimated coefficients

\section{CONCLUSION}

The study indicates that leader's emotional intelligence is positively related to team innovation performance, the relationship between which is partially mediated by leader-member exchange and team psychological safety.

This study deepens the theoretical comprehension of the relationship between leader's emotional intelligence and team innovation performance, and inspires management practice. It might be a feasible way to improve team innovation performance by choosing leaders with high EI or through cultivation and development of leaders' EI.

\section{REFERENCES}

[1] Duan Jinyun, Tian Xiaoming Wang Xianhui. The influence of emotional intelligence on employee creativity [J]. Science Research Management, 2013, 34(8): 106-114. (In Chinese)

[2] Wang Shuhong, Zheng Pei. Relationship between leader's emotional intelligence and the followers' organizational citizenship behavior and task performance: Taking organization atmosphere as intermediary variable [J]. Technology Economics, 2015, 34(3): 33-37. (In Chinese)

[3] Zhang Huihua, Wang Hui. A meta-analysis of the relationship between individual emotional intelligence and workplace performance $[\mathrm{J}]$. Acta Psychologica Sinica, 2011, 43(2): 188-202. (In Chinese)

[4] Mayer J D, Salovey P. What is emotional intelligence? [C] In Salovey P, Sluyter D. (Eds.). Emotional development and emotional intelligence. Implications for educators. New York: Basic Books, 1997: 3-31.

[5] Graen G B, Unl-Bien M. Relationship-based approach to leadership: Development of leader-member exchange (LMX) theory of leadership over 25 years: Applying a multi-level multi-domain perspective [J]. The Leadership Quarterly, 1995, 6(2): 1995-247.

[6] Edmondson A C. Psychological safety and learning behavior in work teams [J]. Administrative Science Quarterly, 1999, 44(2): 350-383.

[7] Han Yi, Liao Jianqiao, Long Lirong. Model of development and empirical study on employee job performance construct [J]. Journal of Management Sciences in China, 2007, 10(5): 62-77. (In Chinese)

[8] Goleman D. What Makes a Leader? [J]. Harvard Business Review, 1998 76(2): 93-102.

[9] Liu Xiaoyu, Sun Jianmin, Zhou Yu. The contingency influence mechanism of transformational/transactional leadership on workgroup innovation performance: the moderating role of workgroup emotional climate [J]. Chinese Journal of Management, 2011, 8(6): 857-864. (In Chinese)

[10] Zhou J, George J M. Awakening employee creativity: the role of leader emotional intelligence [J]. The Leadership Quarterly, 2003, 14: 545-568.

[11] Lee L T-S, Sukoco B M. Reflexivity, stress, and unlearning in the new product development team: the moderating effect of procedural justice [J]. R\&D Management, 2011, 41(4): 410-423.

[12] Hülsheger U R, Anderson N and Salgado J F. Team-level predictor of innovation at work: a comprehensive Meta-analysis spanning three decades of research [J]. Journal of Applied Psychology, 2009, 94(5): $1128-1145$.

[13] Yang Fu, Zhang Lihua. The impact of cognitive style on innovative behavior: The moderating role of team psychological safety and work unit structure [J]. Nankai Business Review, 2012, 15(5): 13-25. (In Chinese)

[14] Liden R C, Maslyn J M. Multidimensionality of leader-member exchange: An empirical assessment through scale development [J]. Original Research Article Journal of Management, 1998, 24(1): 43-72.

[15] Guo Wei, Li Yanping, Du Sheng, Tao Houyong. Authentic Leadership and Employee-team Creativity: A Multi-level Study [J]. Nankai Business Review, 2012, 15(3): 51-60. (In Chinese) 